\title{
ANALYTICAL AND NUMERICAL STUDY OF TUBE DRAWING THROUGH A CONICAL DIE
}

\author{
Sarah Abdul kadhim ${ }^{1}$ and Alaa M. Al-Jassani² \\ ${ }^{1}$ M.Sc. Student, Department of Mechanical Engineering, Faculty of Engineering, \\ University of Kufa, Al-Najaf, Iraq, Email: sarahabdulkadhm@gmail.com \\ ${ }^{2}$ Prof., KCASE, Department of Mechanical Engineering, Faculty of Engineering, \\ University of Kufa, Al-Najaf, Iraq, Email: alaa.aljasani@uokufa.edu.iq

\section{HTTPS://DOI.ORG/10.30572/2018/KJE/120402}

\begin{abstract}
Tube drawing are one of the most important forming processes manufacturing whole board. This process suffers from many problems regarding the deformation behavior of the tube material, as there is no control on the inner radius, i.e. wall thickening and failure at the end. This research involved implementing analytical and numerical simulation to investigate the ability of the tube to resist the failure under applied forces. The mathematical analysis was performed using Tresca yield criterion and the basis of force equilibrium to a representative element in the deformation zone, as it being drawn into the die under quasi-static equilibrium. Explicit dynamic analysis to the tube drawing process was performed using ANSYS 19.2 Workbench program. The analytical solution was used to validate the finite element results obtained from ANSYS program. Two types of material used in this analysis. The die material is made by 304 stainless steel. However, AL2024T351 was selected as tube material. The developed longitudinal stress and the thickness strain from drawing process of the tube through conical die are studied. Investigated parameters that can affect the deformation behavior are taken into account. Such as, the die semi-angle $(\alpha)$ and the die-tube friction coefficient $(\mu)$. It can be observed the very small die semi-angle for all values of die- coefficient of friction leads to a high contact length between the die and tube and this cause the longitudinal stress increase. On the other hand, there are increase in thickness strain with increase in die semi-angle. Finally, it is found that a good agreement between the analytically and the numerically (ANSYS) solutions in the overall trend for all results obtained, stresses and strains.
\end{abstract}

KEYWORDS: Tube drawing; Tube sinking: Analysis; FEA. 


\section{INTRODUCTION}

Tubes, especially those having little diameters and requiring operation just of their external surfaces, are created from drawing coils on machines that fix the stock and slice it to required lengths. Similarly, as with bars, in any case, most tubes are created from straight lengths instead of coiled stock (ASM, 2010). The main advantage of tube drawing process to reduce the diameters, develop dimensional accuracy and refine surface finish (Dieter et al., 2003). The tube is fed into the die and pulled by external load to take the desired dimensions Fig. 1 with no need of mandrel. The outer diameter is decreased while the length and wall thickness increases.

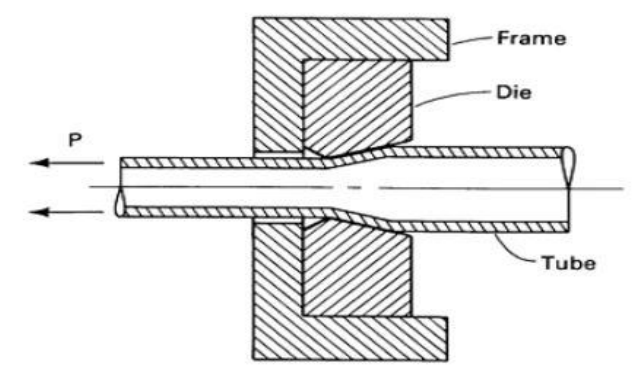

Fig. 1. Tube drawing without a mandrel (tube sinking).

Reducing operational cost is one of the advantages of the sinking tube process. The process does not require sophisticated equipment. The operation must be done carefully and it is not desired to apply excessive load because applying an excessive load can lead to failure of the process (Shaheen, 2007). The value of the increase in tube length and thickness relies on many factors. These factors include die geometry, flow stress of drawn material, and friction between the drawn part and die.

Yang et al., (2008), studied the drawing process using a numerical analysis on a hollow tube made of copper. Many parameters affecting drawing process have been studied. The results obtained for the semi angle assist the researchers to decide the best design of the die and prolong the life of the die. An experimental work was carried out in (Tang et al., 2009) to examine the plastic deformation of the tube's teeth and the groove area. It was observed that the highest stress appeared at the grooves and the highest damage occurred at the tip of the teeth. (Béland et al., 2011), measured the deformation and the reaction force between the mandrel and the die. An optimization study was carried out to minimize the longitudinal stresses. The effect of the coefficient of friction on the distribution of von-Mises stresses, equivalent plastic strain and axial stresses was investigated by (Kumar and Agnihotri, 2013). It was observed that the vonMises and longitudinal stresses as increases with increasing the value of friction coefficient. 
(Lin et al., 2014), built a model of low carbon steel using finite element software to investigate the sinking drawing process of the tube. The effect of different parameters such as the length and semi-angle of the secondary shrinking region has been studied to select the best design of the die. (Halaczek, 2016), studied the production of tubes drawing process. The tubes were assumed to be made of non-ferrous metals, bimetallic, and bimetallic tubes in layer's composition: copper-brass and brass-copper. Different compositions of the tube materials with different wall thickness have been studied. It was concluded that it is hard to anticipate the material response in the drawing die of the bimetallic tubes manufactured by sinking drawing process. (Boutenel et al., 2018), developed a finite element model to anticipate the desired dimension of a tube manufactured by cold drawing. An experimental analysis was also carried out to validate the finite element results. It was shown that the drawing force depends on the semi die angle. The relative thickness influences the mechanical performance of the tube during the drawing process. (Chobaut et al., 2019), experimental and finite element analysis were followed to investigate the drawing process extensively. The friction coefficient between the die and the specimen was estimated by the finite element model and experiment. The obtained results revealed that using the lubrication led to reduce the coefficient of friction and prohibit sticking and slipping of the specimen. A recent study was done by (Gattmah et al., 2020), to compute the residual hoop and axial stresses developed inside the tube wall of AISI steel 1010 by using removing layer method. An explicit dynamic- numerical solution was performed to anticipate the developed stresses. Then, the numerical and experimental results are validated with Xray diffraction results. A good match between numerical and experimental results has been obtained.

\section{MATHEMATICAL SOLUTION}

The mathematical analysis of tube drawing has been implemented inside the conical die to control the outer diameter. The mathematical analysis of tube drawing process is a complicated process as it includes many variables (Ling, 2006). These variables involve geometric shape of the sample, the shape of the die, and the conditions of friction (ASM, 2010). A tube of circular cross-section and uniform thickness is drawn through a conical die so that its original radius is $r_{o}$ reduced to a final radius $r_{f}$ with a semi die angle $\alpha^{\circ}$ as see in Fig. 2-a. 

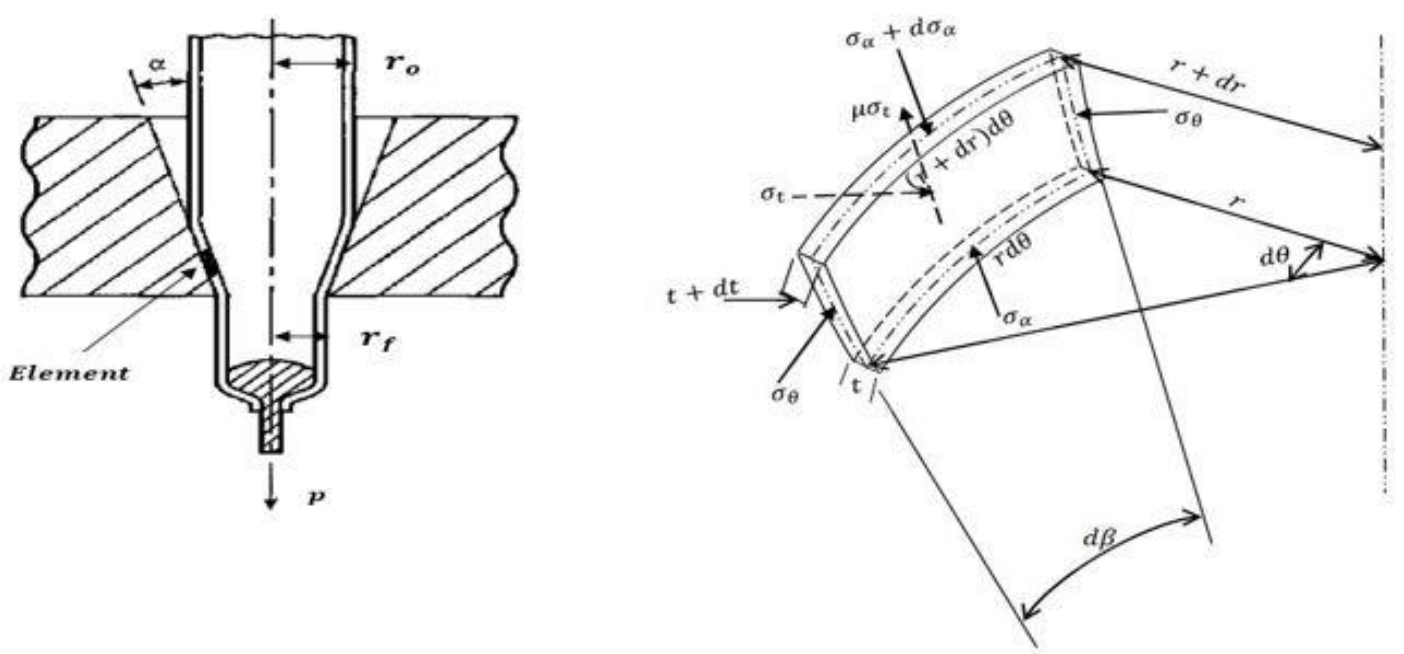

Fig. 2. (a) Tube drawing through a conical die (Ling, 2006). (b)Tube wall element in the deformation zone.

Also, it is assumed that thickness to diameter ratio very small, i.e. the drawing process are dealt with thin-walled tube forming. If the tube is sufficiently long, the problem may be considered as one of steady state for the calculation of stress and strains during the drawing (Bhaduri , 2018; G. S. and W. M. B. Jr, ) 15]. The analysis is carried out by considering a representative element of the tube as shown in Fig. 2-b.

Resolving the forces perpendicular to the die face, to get:

$\sigma_{t}\left(\frac{d r}{\sin \alpha}\right)((r+t) d \theta)-2 \sigma_{\theta}(\cos \alpha)\left(\sin \frac{d \theta}{2}\right)\left(\frac{d r}{\sin \alpha}\right)(t)=0$

Since $d \theta$ is small, then; $\sin \frac{d \theta}{2} \approx\left(\frac{d \theta}{2}\right)$ and the equation become;

$\sigma_{t}=\sigma_{\theta}(\cos \alpha)\left(\frac{t}{r+t}\right)$

Resolving the forces in the direction of $\sigma_{\alpha}$, to get:

$\left(\sigma_{\alpha}+d \sigma_{\alpha}\right)(r+d r)(t+d t) d \theta-\left(\sigma_{\alpha}\right)(r)(t) d \theta+$

$\mu \sigma_{t}\left(\frac{d r}{\sin \alpha}\right)(r+t) d \theta+2 \sigma_{\theta}\left(\frac{d r}{\sin \alpha}\right)(t)(\sin \alpha)\left(\sin \frac{d \theta}{2}\right)=0$

Simplify and substituting for $\sigma_{t}$ from equation (1) into equation (2) to get:

$\frac{d\left(\sigma_{\alpha} r t\right)}{d r}+\sigma_{\theta} t(1+\mu \cot \alpha)=0$ 
To determine hoop stress, it might be suitable to consider the material under consideration to be rigid-work hardening, and the modified Tresca yield criteria take the form (Boutenel et al., 2018):

$\sigma_{\alpha}+\sigma_{\theta}=m Y\left[1+k\left(\ln \frac{r_{o}}{r}\right)^{n}\right]$

Substitute for $\sigma_{\theta}$ from equation (4) into equation (3) to get:

$\frac{d \sigma_{\alpha}}{d r}=-\frac{1}{r}(1+\mu \cot \alpha) m Y\left[1+k\left(\ln \frac{r_{o}}{r}\right)^{n}\right]+\frac{\mu \cot \alpha}{r} \sigma_{\alpha}$

Now, it is possible to find the longitudinal stress $\sigma_{\alpha}$ at any radius $r$ for the variables in equation (5), and considering the boundary condition where $\sigma_{\alpha}=0$ at die inlet $r=r_{o}$, as it is assumed no back-pull at entry to the die.

To determine $\varepsilon_{\alpha}$ use the flow rule, Levy-Mises relation as (Bhaduri, 2018; Sachs and Baldwin, 1946):

$\frac{d \varepsilon_{t}}{d \varepsilon_{\theta}}=\frac{\frac{d t}{t}}{\frac{d r}{r}}=\frac{\sigma_{t}-\frac{1}{2}\left(\sigma_{\theta}+\sigma_{\alpha}\right)}{\sigma_{\theta}-\frac{1}{2}\left(\sigma_{\alpha}+\sigma_{t}\right)}=\frac{2 \sigma_{t}-\left(\sigma_{\theta}+\sigma_{\alpha}\right)}{2 \sigma_{\theta}-\left(\sigma_{\theta}+\sigma_{t}\right)}$

The thickness stress given by equation (1) and substitute for $\sigma_{\theta}$ from equation (4) to get:

$\frac{d t}{d r}=\frac{t}{r}\left[\frac{2 \sigma_{\alpha}-m Y\left[1+k\left(\ln \frac{r_{o}}{r}\right)^{n}\right]}{2 m Y\left[1+k\left(\ln \frac{r_{o}}{r}\right)^{n}\right]-\sigma_{\alpha}}\right]$

Substituting the value of $\sigma_{\alpha}$ at any radius $r$ found from equation (5) into equation (7) to determine the instantaneous value of the wall thickness $t$ at that position in the deformation zone. Hence the thickness strain $\varepsilon_{t}$ are obtained.

Using the principle of volume constancy as (ASM, 2010 ; Ling, 2006):

$\varepsilon_{\alpha}+\varepsilon_{t}+\varepsilon_{\theta}=0$

Then by knowing the hoop strain from equation $\varepsilon_{\theta}=\ln \frac{r_{o}}{r_{f}}$ and thickness strain $\varepsilon_{t}$ from equation (7), then it is easy to compute the value of the longitudinal strain $\varepsilon_{\alpha}$ at any radius $r$.

\section{NUMERICAL WORK}

It is very important to validate the results of tube drawing process that obtained through analytical solutions. The simulation of tube drawing process through a die was done using ANSYS Workbench 19.2. In this analysis, the outer and inner diameters of the tube are reduced 
while the thickness is kept the same. During this process, set of diameter range, coefficient of friction and die angle are selected to investigate their effects on developed stress and strain.

In the present work the die material is made by 304 stainless steel. However, AL2024T351 was selected as tube material as shown in Table 1 (ASM, 2020).

Table 1. Mechanical properties of 304 Stainless Steel and AL2024T351 (ASM, 2020).

\begin{tabular}{ccc}
\hline Properties & 304 S Steel & AL2024T351 \\
\hline Density & $7750 \mathrm{Kg} / \mathrm{m}^{3}$ & $2780 \mathrm{Kg} / \mathrm{m}^{3}$ \\
Ultimate Tensile Strength & $586 \mathrm{MPa}$ & $469 \mathrm{MPa}$ \\
Tensile Yield Strength & $210 \mathrm{MPa}$ & $324 \mathrm{MPa}$ \\
Modulus of Elasticity & $193 \mathrm{GPa}$ & $73.1 \mathrm{GPa}$ \\
Poisson's Ratio & 0.31 & 0.33
\end{tabular}

Quality of meshing depends on element size. The element size was taken 0.0005 for the tube and the die structure, great care was taken in meshing this model, quadratic element was used for good accuracy in stress analysis. The Quadratic quadrilateral element was used in this model. This type of element used in two-dimensional problem because it gives flexibility in modeling and good accuracy analysis (Chen and Liu, 2014). This element contains eight nodes in order to tolerate irregular shapes without much loss of accuracy Fig. 3.

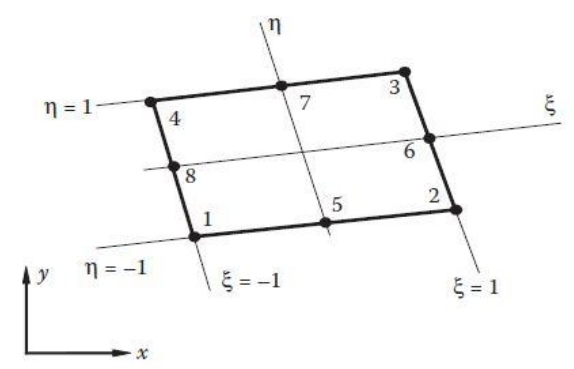

Fig. 3. Quadratic quadrilateral element.

In ANSYS program, axisymmetric analysis was performed to find the stress and the deformation in two-dimensional plasticity tube drawing process using FEA. Fig. 4 shows the

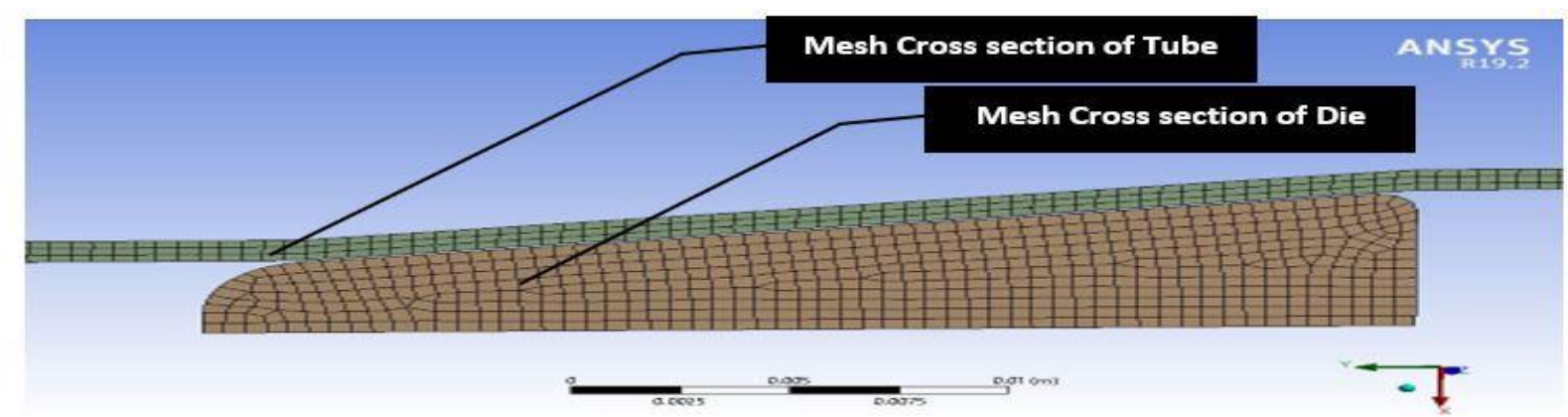


Fig. 4. Symmetric cross section of tube and die about $y$ axis.

\section{RESULTS ANALYSIS AND DISCUSSIONS}

The results obtained from performing analytical is presented and discussed. The die friction condition and the die geometry were determined toward the selection of the best and the practical values of the coefficient of friction and the die semi-angle for the conical die used in the research.

The effect of the die-tube coefficient of friction for values ranging from (0 to 0.1$)$ was determined. This trend in Fig. 5 was estimated for different values of die semi-angle where the increase in coefficient of friction resulted in an increase in longitudinal stress. The aforementioned agree with (Gattmah et al., 2017; Boutenel and Delhomme, 2018).

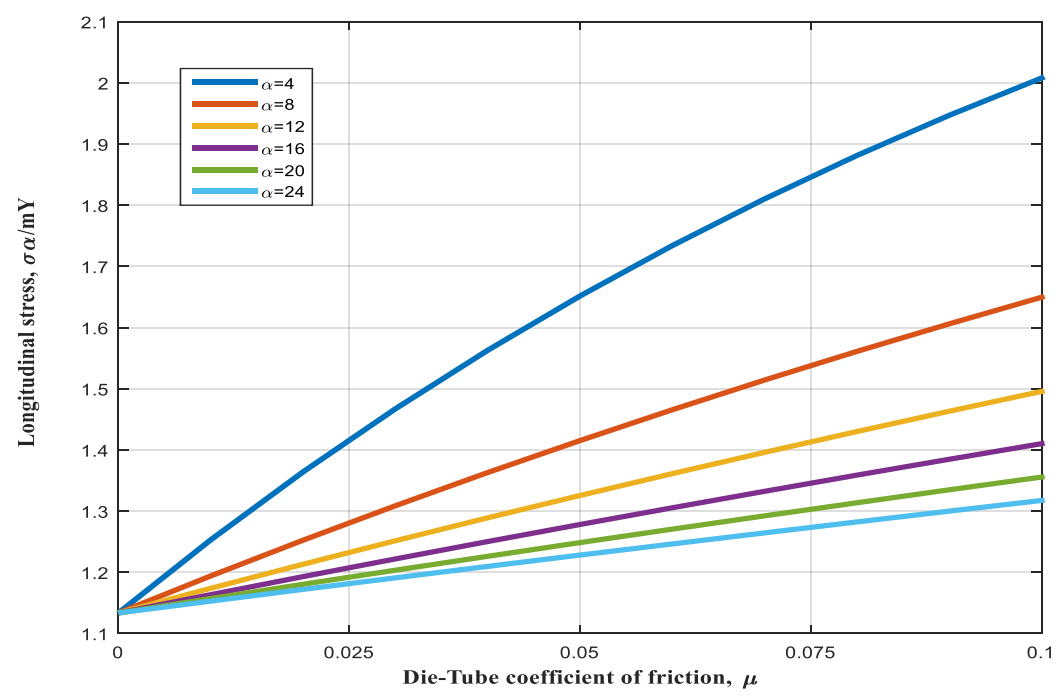

Fig. 5. Effect of coefficient of friction on the longitudinal stress for, $R=0.4, \frac{t_{o}}{r_{o}}=0.05, n=$ 0.28 and $k=2$.

Very small die semi-angle leads to a high contact length between the die and the tube. This causes high friction losses. In other hand, very high semi-die angle contributes to a velocity discontinuity which in return leads to high predominant factor. Therefore, selecting an appropriate die semi-angle is necessary to minimize the operational required stress. 
In order to study the effect of die semi -angle on the thickness strain, Fig. 6 shows this relation. It can be clearly seen that the thickness strain remains constant when $\mu$ is equal to zero. The thickness strain gets higher when the die semi-angle increases, i.e., the high value of $\alpha$ produces high thickness strain. This behavior can be explained and attributed to the complex relation of the coefficient of friction between the die and the tube and the die semi-angle, as mentioned earlier.

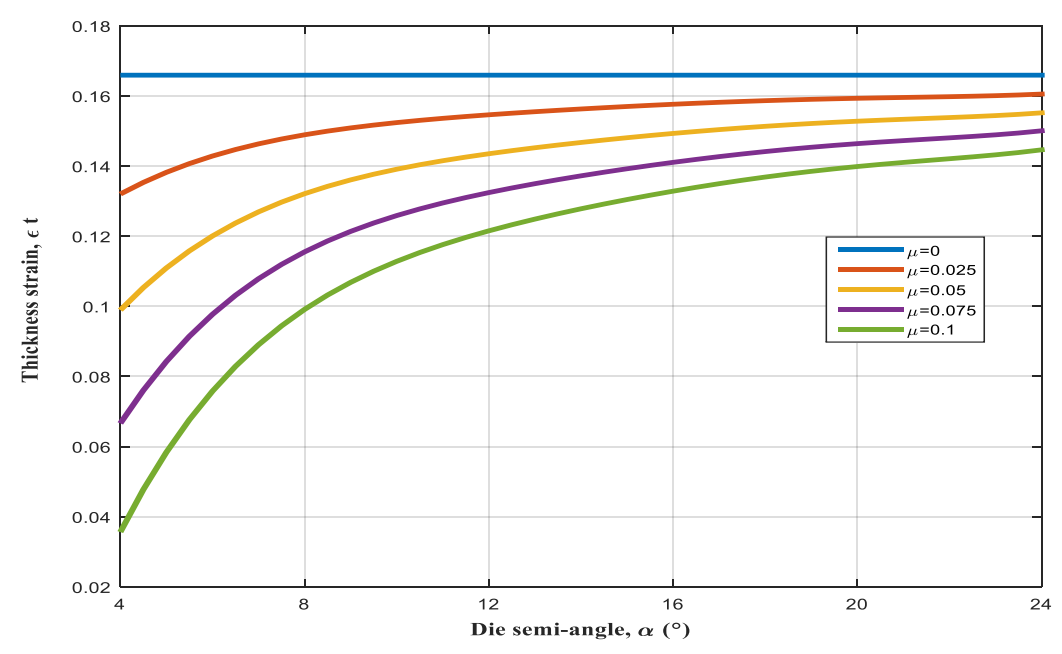

Fig. 6. Effect of die semi-angle on the thickness strain for , $R=0.4, \frac{t_{o}}{r_{o}}=0.05, n=$ 0.28 and $k=2$

In this work, the results obtained for the longitudinal and thickness stresses and strains distribution through drawing process are represented and discussed. The cases selected for study involved estimating the stresses and strains (longitudinal and thickness) at die-semi angle $\left(\alpha=10^{\circ}\right)$, friction coefficient $(\mu=0.05)$, hardening exponent $(n=0.28)$ and hardening constant $(k=2)$ as can be shown in Figs 7, 8, 9 and 10 respectively. It can be noted that the results viewed as contour distribution through a path start from inlet to the outlet radius.

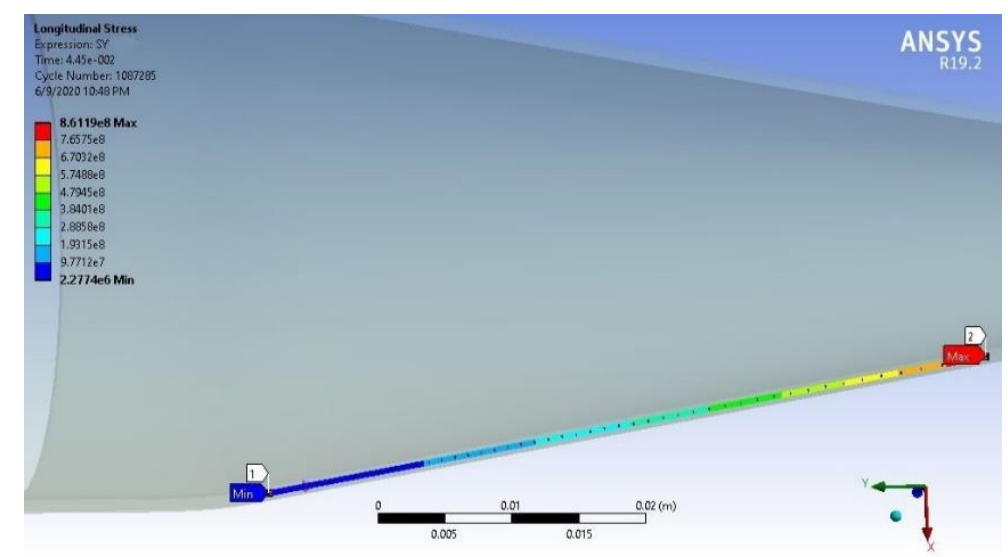


Fig. 7. The results viewing of longitudinal stress.

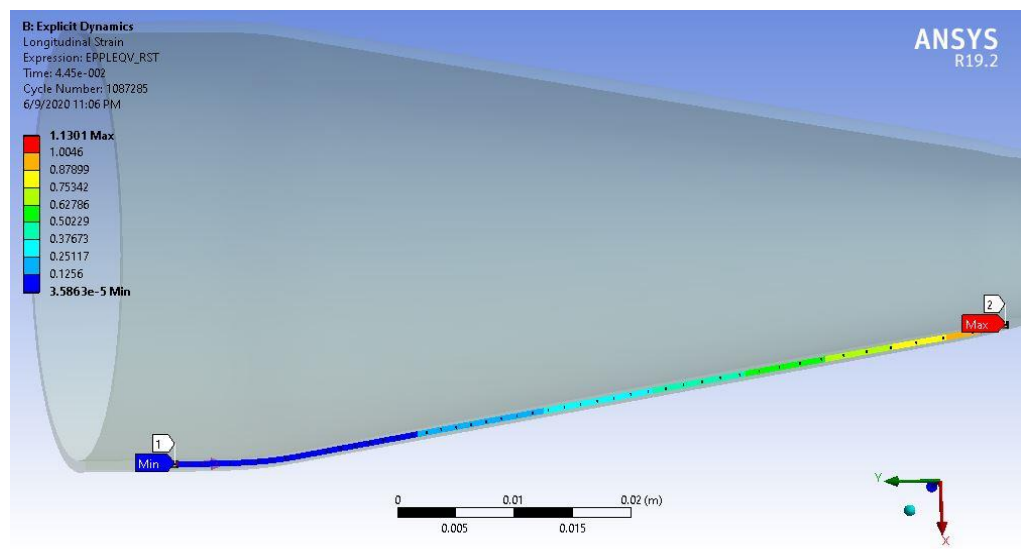

Fig. 8. The results viewing of longitudinal strain.

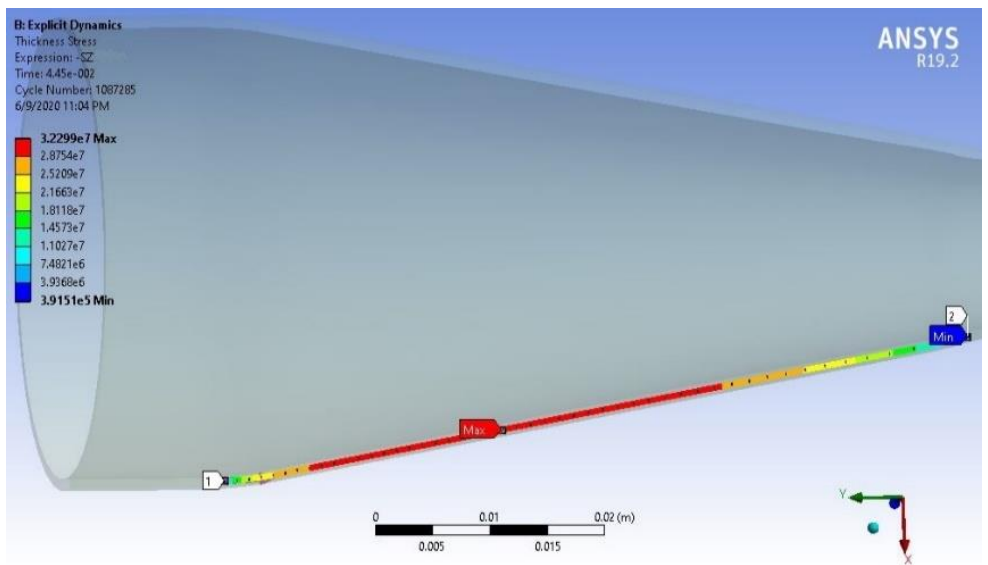

Fig. 9. The results viewing of thickness stress.

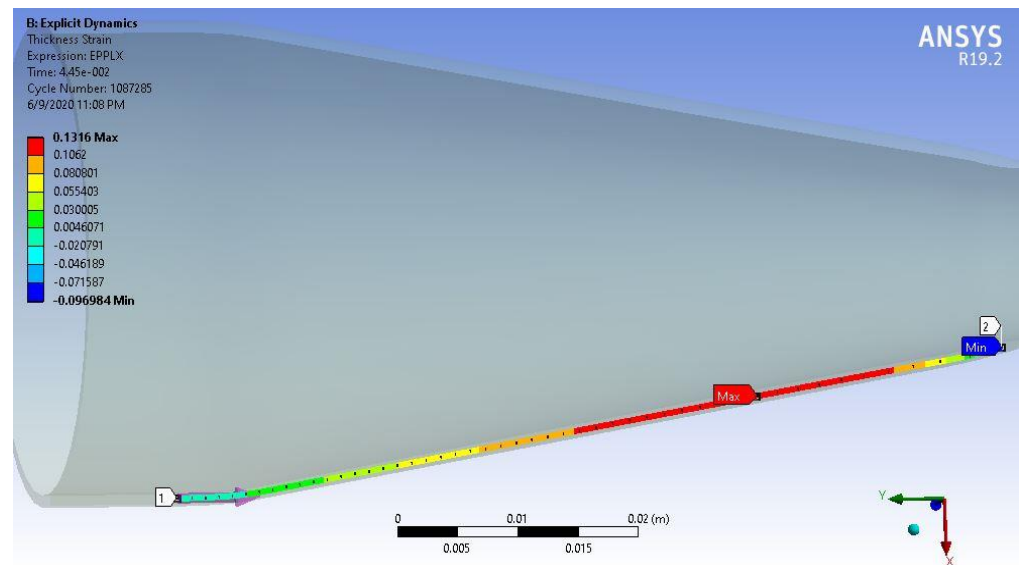

Fig. 10. The results viewing of thickness strain. 
The comparison between analytical and ANSYS was performed. Fig. 11 demonstrates the comparison between the longitudinal stress conducted using numerical and analytical modeling. It can be seen from Fig. 11 that the longitudinal stress increases with the position toward exit. This behavior is due to the increase in energy consumption at the inlet and the outlet for the forming area and the required plastic deformation at the end. This increase agrees with these research articles (ASM, 2021; Chen and Liu, 2017; Lee, 2017).

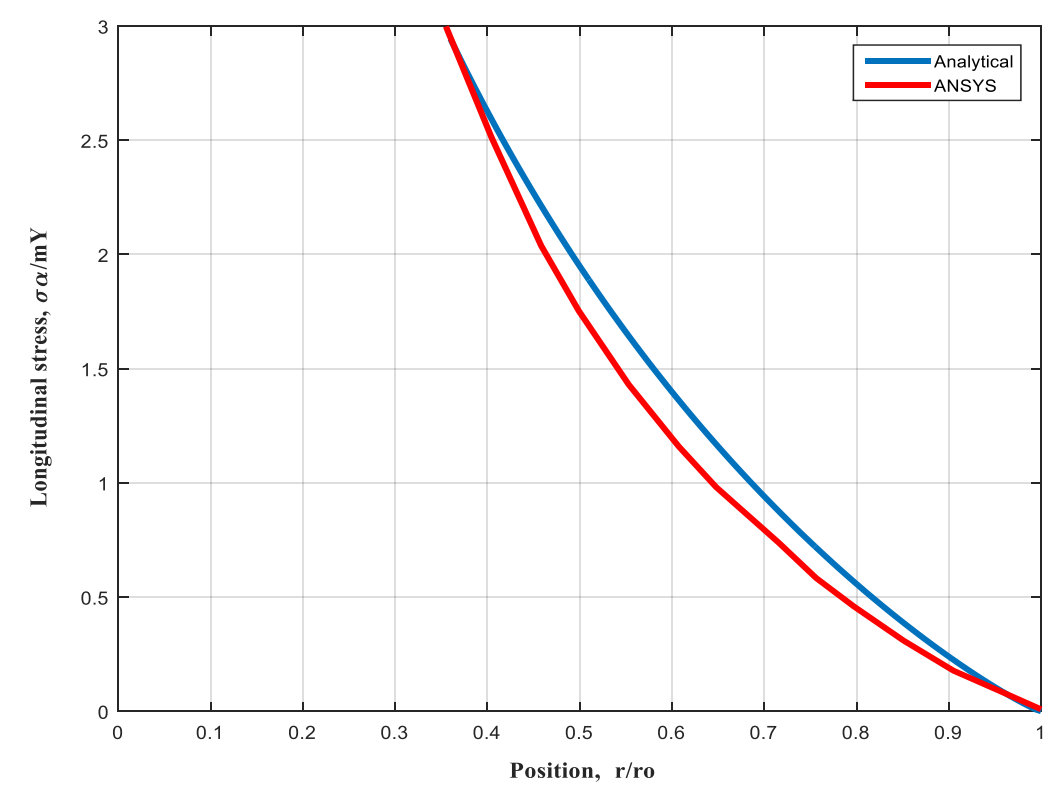

Fig. 11. Distribution of the longitudinal stress along the deformation zone analytical and ANSYS for, $\frac{t_{o}}{r_{o}}=0.05, \mu=0.05, \alpha=10^{\circ}, n=0.28$ and $k=2$.

Fig. 12 represents the distribution of the thickness stress with the position ratio. Both friction and strain hardening have substantial effect on $\sigma_{t}$ (die pressure ratio). Strain hardening rate is necessary to cause the die pressure to increase with the reduction in radius. However, in the exist direction, the friction component and perpendicular pressure on the thickness of the tube wall cause reduced in die pressure (thickness stress) value (Chen and Liu, 2017). 


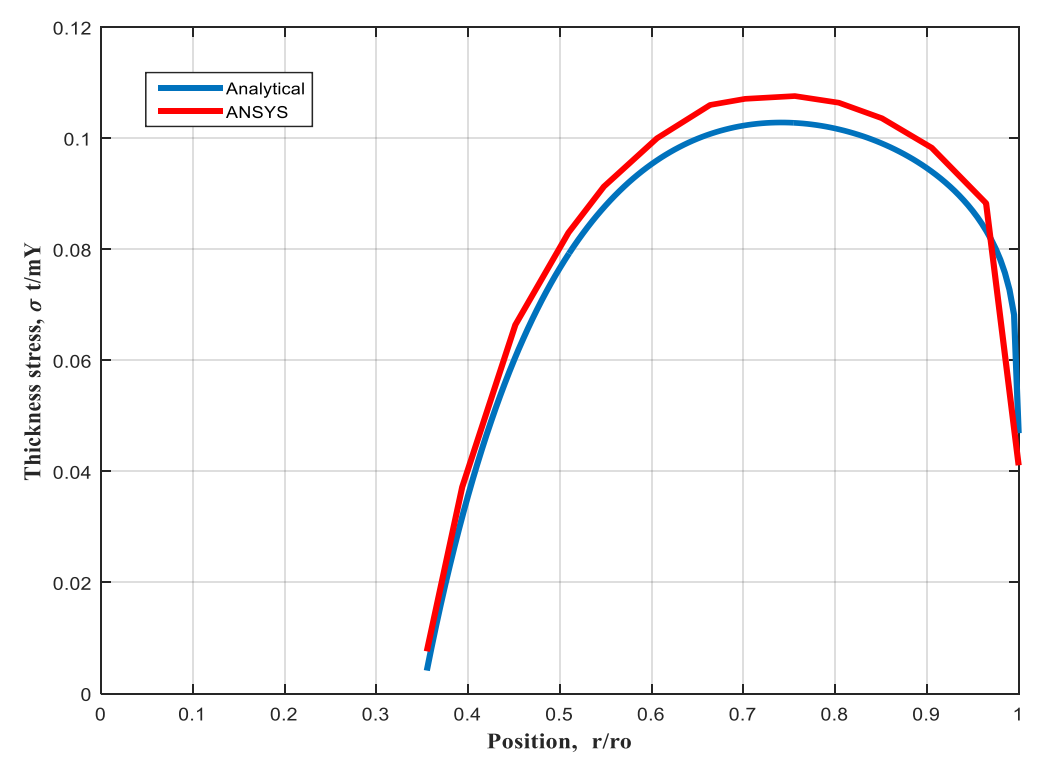

Fig. 12. Distribution of the thickness stress along the deformation zone, analytical and ANSYS

$$
\text { for, } \frac{t_{o}}{r_{o}}=0.05, \mu=0.05, \alpha=10^{\circ}, n=0.28 \text { and } k=2 \text {. }
$$

Fig. 13 presents the longitudinal strain that is computed using analytical and ANSYS approaches. The longitudinal strain increases with decreasing of the tube radius, starting from zero at inlet section to a maximum value at exit position. This behavior can be explained according to the principle of volume constancy in equations 8 .

The discrepancy in thickness strain calculated as shown in Fig. 14. The thickness strain varies with the position, at which it increases to some extent (thickening), after that begin decreasing (thinning) gradually until it reached at minimum values at exist point. The main conclusion that can be drawn from this figure is that the high reductions in strain hardening substantially increases reduction in thickness strain. 


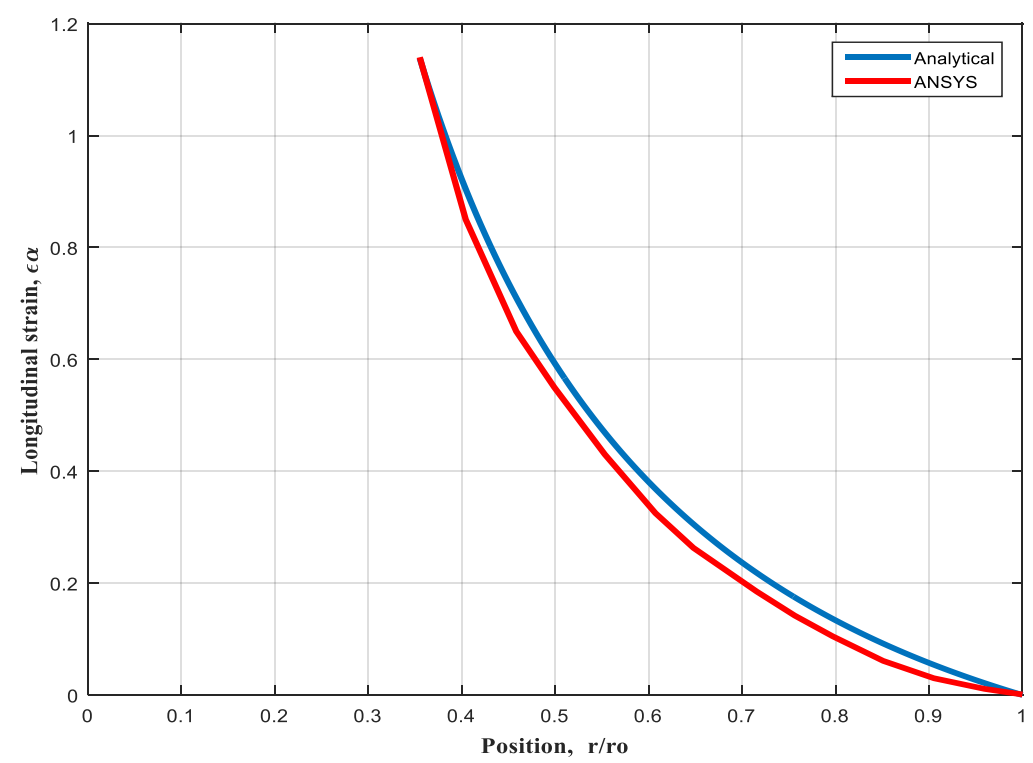

Fig. 13. Distribution of the longitudinal strain along the deformation zone, analytical and ANSYS for, $\frac{t_{o}}{r_{o}}=0.05, \mu=0.05, \alpha=10^{\circ}, n=0.28$ and $k=2$.

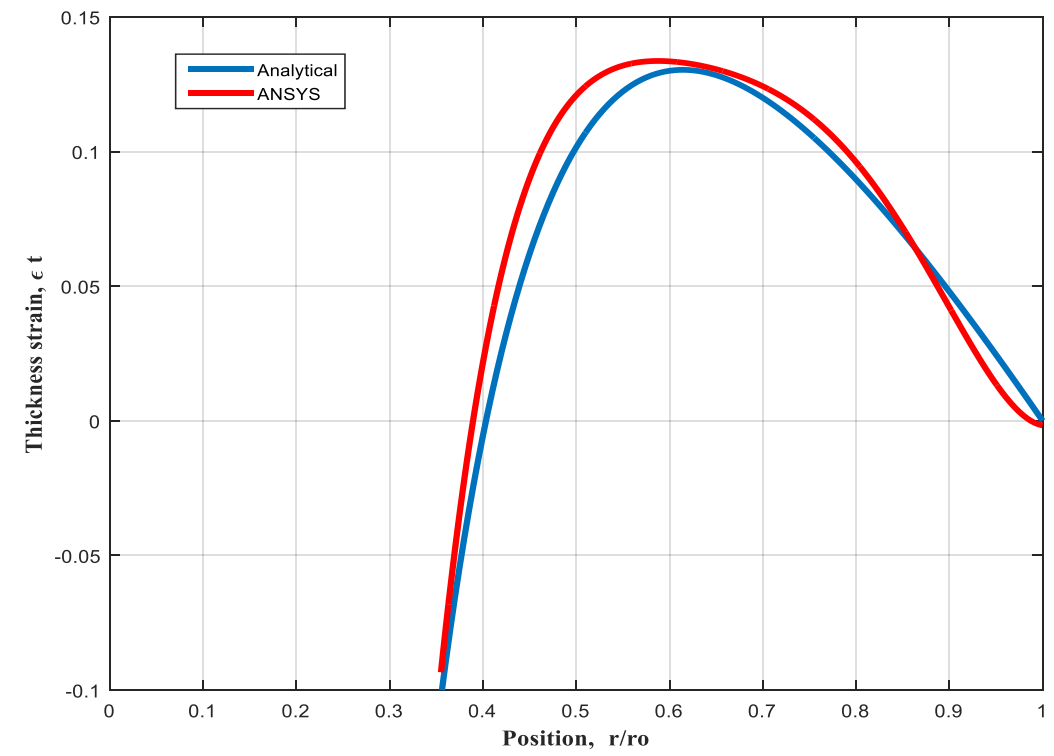

Fig. 14. Distribution of the thickness strain along the deformation zone, analytical and ANSYS for, $\frac{t_{o}}{r_{o}}=0.05, \mu=0.05, \alpha=10^{\circ}, n=0.28$ and $k=2$.

\section{CONCLUSIONS}

It can be concluded that the analytically and the numerically (ANSYS) solutions has a fair agreement between them. There is an interaction and non-linearity in the relation between the die- coefficient of friction $(\mu)$ and die semi-angle $(\alpha)$ and the effects on the stresses and strains. For example, at $\alpha=4^{\circ}$, the effect of $\mu$ are more pronounced in increasing the longitudinal stress 
and decreasing the thickness strain. But this effect at higher values of ( $\alpha=24^{\circ}$ for example), will be decreased in non-linear relation.

\section{REFERENCES}

ASM, “Asm Hand Book Forming and Forigng,” p. 2110, 2010.

G. E. Dieter, H. A. Kuhn, and S. L. Semiatin, "Handbook Workability of Process Design," 2003.

L. Shaheen, “Tube Drawing.,” Tube Pipe J., pp. 126-131, 2007.

X. J. Yang, F. H. Sun, Z. M. Zhang, H. S. Shen, and S. S. Guo, "Optimization of drawing parameters for copper tubes with hollow sinking based on FEM simulation," Chinese J. Nonferrous Met., vol. 18, no. 12, pp. 2245-2252, 2008.

Y. Tang, L. sheng Lu, D. Yuan, Q. hui Wang, and X. lin Zhao, "Experimental and FEM study on sinking of miniature inner grooved copper tube,” J. Mater. Process. Technol., vol. 209, no. 12-13, pp. 5333-5340, 2009.

J. F. Béland, M. Fafard, A. Rahem, G. D’Amours, and T. Côté, "Optimization on the cold drawing process of 6063 aluminium tubes,” Appl. Math. Model., vol. 35, no. 11, pp. 5302$5313,2011$.

P. Kumar and G. Agnihotri, "Study of Seamless Tube Cold Drawing Using Finite Element Simulation," vol. 1, pp. 1-6, 2013.

Z. Lin, B. Shen, F. Sun, Z. Zhang, and S. Guo, "Diamond-coated tube drawing die optimization using finite element model simulation and response surface methodology," Proc. Inst. Mech. Eng. Manuf., vol. 228, no. 11, pp. 1432-1441, 2014.

D. Halaczek, "Analysis of manufacturing bimetallic tubes by the cold drawing process," Arch. Metall. Mater., vol. 61, no. 1, pp. 241-248, 2016.

F. Boutenel, M. Delhomme, V. Velay, and R. Boman, "Finite element modelling of cold drawing for high-precision tubes," Comptes Rendus - Mec., vol. 346, no. 8, pp. 665-677, 2018. N. Chobaut et al., "Miniaturized tube fixed plug drawing: Determination of the friction coefficients and drawing limit of 316 LVM stainless steel," J. Mater. Process. Technol., vol. 263, pp. 396-407, 2019.

J. Gattmah, F. Ozturk, and S. Orhan, "A new development of measurement technique for residual stresses generated by the cold tube drawing process with a fixed mandrel," Int. J. Adv. 
Manuf. Technol., vol. 108, no. 11-12, pp. 3675-3687, 2020.

Ling, F. F., "Mechanical Engineering Series: Applied Plasticity,”2006.

Bhaduri, Amit, "Mechanical Properties and Working of Metals and Alloys," Springer Series in Materials Science, 2018.

Sachs, G., and Baldwin, W. M., Jr., "Stress Analysis of Tube Sinking," TRANS. ASME, Vol. 68,1946, p. 655 .

“ASM Aerospace specification Metals Inc ,” http://asm.matweb.com.

X. Chen and Y. Liu, "Finite element modeling and simulation with ANSYS workbench," CRC Press. 2014.

J. Gattmah, F. Ozturk, and S. Orhan, "Effect of the process parameters on cold tube drawing with a fixed plug using finite element analysis for AISI 1010 steel ABSTRACT," Sak. Univ. J. Sci., vol. 21, no. 5, pp. 886-892, 2017.

F. Boutenel, M. Delhomme, V. Velay, and R. Boman, "Finite element modelling of cold drawing for high-precision tubes," Comptes Rendus - Mec., vol. 346, no. 8, pp. 665-677, 2018. H. H. Lee, "Finite Element Simulations with ANSYS Workbench 17”. SCD Publications, 2017. 